\title{
Autoconcepto, autoestima, motivación y su influencia en el desempeño académico. Caso: alumnos de la carrera de Contador Público
}

Self-concept, self-esteem, motivation and its influence on academic performance. Case: students of the public accounting program

Autoconceito, autoestima, motivação e sua influência no desempenho escolar. Caso: alunos contadores públicos

José Ángel Pendones Fernández

Instituto Tecnológico Superior de Nuevo Casas Grandes, México

jpendones@itsncg.edu.mx https://orcid.org/0000-0001-6993-1995

Yolanda Flores Ramírez Instituto Tecnológico Superior de Nuevo Casas Grandes, México yflores@itsncg.edu.mx https://orcid.org/0000-0001-9012-9550

Germán Espino Olivas Instituto Tecnológico Superior de Nuevo Casas Grandes, México gespino@itsncg.edu.mx https://orcid.org/0000-0002-6067-5662

Félix Arnoldo Durán Núñez Instituto Tecnológico Superior de Nuevo Casas Grandes, México fduran@itsncg.edu.mx https://orcid.org/0000-0001-9998-8781 


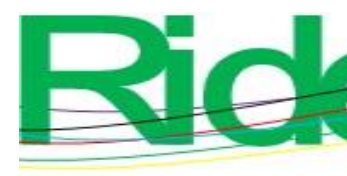

Revista Iberoamericana para la Investigación y el Desarrollo Educativo ISSN $2007-7467$

\section{Resumen}

El propósito de este estudio fue analizar la influencia de los factores psicosociales en el desempeño académico, ya que existe un índice muy elevado de deserción y reprobación en el nivel de educación superior. La unidad de análisis estuvo constituida por los alumnos de la carrera de Contador Público, a quienes se les aplicó una encuesta con tres escalas con un coeficiente de validez aceptable. Con la información obtenida se generó una matriz para observar si existían diferencias estadísticamente significativas entre hombres y mujeres. Se identificó como variable dependiente el desempeño académico tomando como criterio un nivel de significancia estadística $\mathrm{p}<0.05$, utilizando la prueba t de Student para dos muestras independientes. Los resultados muestran que en las mujeres hay una mayor influencia de los factores motivación, autoestima y autoconcepto; en cuanto a los hombres, el factor con mayor influencia es autoestima, y entre más bajas puntuaciones obtienen en estos rubros menor es su desempeño académico. También se obtuvo una ecuación por medio de un modelo de regresión para estimar la variable de respuesta desempeño académico, apoyado en la variable de predicción autoconcepto. En conclusión, se puede indicar que autoestima, autoconcepto y motivación son factores que se interrelacionan en el proceso de aprendizaje, y que los alumnos que poseen fortaleza psicológica tienen un mayor rendimiento académico.

Palabras clave: desempeño académico, identidad, motivación, personalidad, psicología.

\section{Abstract}

The purpose of this study is to analyze whether psychosocial factors influence academic performance, since there is a very high dropout and failure rate at the higher education level. The unit of analysis were the students of the Public Accountant Program to whom a three scales survey with an acceptable validity coefficient was applied. With the information obtained, a matrix was generated to observe if there are statistically significant differences between men and women. Academic performance was identified as a dependent variable, taking as a criterion a level of statistical significance $\mathrm{p}<0.05$, using the student's " $\mathrm{t}$ " test for two independent samples. The results show that in women there is a greater influence of the factors: motivation, self-esteem and self-concept; As for men, the factor with the greatest influence is self-esteem, the lower scores they obtain in these areas, the lower their academic performance. An equation was also obtained through a regression model to estimate the 


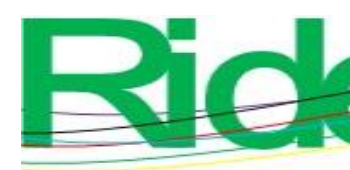

\section{Revista Iberoamericana para la Investigación y el Desarrollo Educativo ISSN $2007-7467$}

response variable academic performance, supported by the self-concept predictor variable. Conclusion: with the measurement it is determined that self-esteem, self-concept and motivation are factors that are interrelated in the learning process, students who have psychological strength have a higher academic performance.

Keywords: academic performance, identity, motivation, personality, psychology.

\section{Resumo}

O objetivo deste estudo foi analisar a influência dos fatores psicossociais no desempenho escolar, visto que existe uma taxa de evasão e reprovação muito elevada no ensino superior. A unidade de análise foi constituída pelos alunos da carreira de Contador Público, aos quais foi aplicada uma enquete com três escalas com coeficiente de validade aceitável. Com as informações obtidas, foi gerada uma matriz para observar se havia diferenças estatisticamente significativas entre homens e mulheres. O desempenho acadêmico foi identificado como variável dependente, tomando-se como critério um nível de significância estatística $\mathrm{p}<0,05$, utilizando o teste $\mathrm{t}$ de Student para duas amostras independentes. Os resultados mostram que nas mulheres há maior influência dos fatores motivação, autoestima e autoconceito; Já para os homens, o fator de maior influência é a autoestima, e quanto mais baixos escores nessas áreas, menor é o seu rendimento escolar. Também foi obtida uma equação por meio de um modelo de regressão para estimar o desempenho acadêmico da variável resposta, apoiado na variável preditora de autoconceito. Em conclusão, pode-se indicar que a autoestima, o autoconceito e a motivação são fatores que se inter-relacionam no processo de aprendizagem, e que alunos com força psicológica apresentam melhor desempenho acadêmico.

Palavras-chave: desempenho acadêmico, identidade, motivação, personalidade, psicologia.

Fecha Recepción: Enero 2021

Fecha Aceptación: Julio 2021 


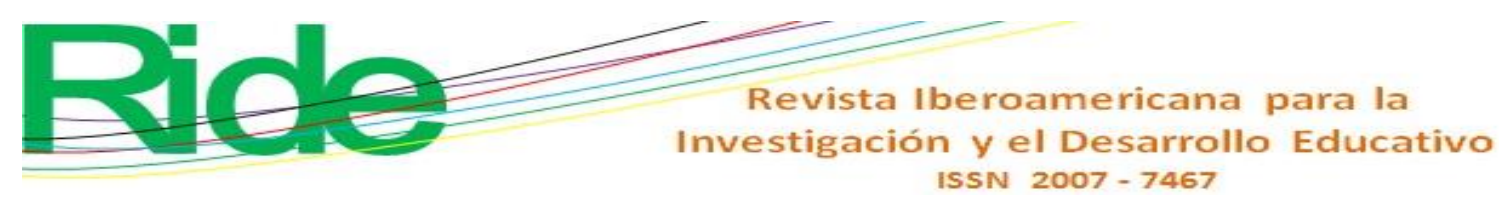

\section{Introducción}

La globalización y los cambios en la estructura económica en la actualidad exigen una mayor preparación académica para acceder a mejores oportunidades. Por esta razón, la educación universitaria se convierte en un reto para los jóvenes desde el momento mismo de la elección de la carrera. Según las estadísticas del sistema educativo nacional (Secretaría de Educación Pública [SEP], 2019), en "México se tiene una matrícula en nivel superior en modalidad escolarizada de 3943544 de los cuales 1980888 son mujeres y 1962656 hombres” (p. 36), aunque "la tasa de eficiencia terminal en jóvenes de entre 25 y 29 años es de apenas 67 \%; además, hay una tasa de abandono superior a 20 \%" (Román, 17 de febrero de 2020, párr. 1). La Asociación Nacional de Universidades e Instituciones de Educación Superior (ANUIES, s. f.) en su anuario del 2018-2019 muestra en el estado de Chihuahua un ingreso de 132960 estudiantes, de los cuales egresan 20612 y se titulan 16166 en total. Estas cifras reflejan un alto porcentaje de deserción, reprobación y una baja eficiencia terminal, de ahí que sea "fundamental encontrar las causas de este problema, ya que en algunos casos se puede tener una capacidad intelectual y aptitudes y sin embargo no estar obteniendo un rendimiento adecuado" (Jiménez, 2000, citado por Edel Navarro, 2003, p. 3).

Estudios realizados sobre el rendimiento académico evidencian que existen múltiples factores que inciden en este fenómeno. La mayoría de las investigaciones realizadas enfocadas en el nivel superior analizan factores cognitivos y socioeconómicos. Gran parte de estos estudios han dejado de lado los factores psicológicos que hacen referencia a todos los constructos relacionados con la salud mental del estudiante y a su personalidad, tales como autopercepción, autoestima, sensación de autoeficacia, autoconcepto, capacidad de adaptación a los cambios, habilidades interpersonales, motivación, entre otros; estos son factores que pueden determinar la reprobación e incluso la deserción del alumno universitario.

Actualmente, la mayoría de los niveles educativos en México se centran en la formación basada en el enfoque por competencias que integra la teoría con la práctica. Este modelo educativo busca el desarrollo del espíritu emprendedor como base del crecimiento personal y del desarrollo socioeconómico, y se fundamenta en la organización curricular con base en proyectos y problemas. Sin embargo, según Tobón (2005), “existen inconsistencias y falta de claridad en la estructura conceptual del término competencias, puede llegar a confundirse con otros conceptos similares tales como inteligencia, funciones, capacidades, calificaciones, 


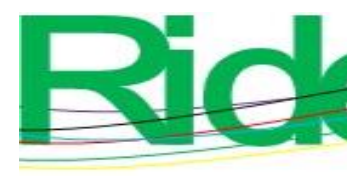

\section{Revista Iberoamericana para la Investigación y el Desarrollo Educativo ISSN $2007-7467$}

habilidades, actitudes, destrezas, indicadores de logro y estándares” (p. 15). Considerando esta estructura conceptual, los docentes suelen impartir los programas académicos tomando en cuenta las habilidades cognitivas del alumno suponiendo que en los niveles previos ya desarrollaron los procesos mentales necesarios para lograr un buen desempeño. En pocas palabras, se centran únicamente en el aspecto intelectual dejando de lado factores internos que pueden afectar el nivel de progreso.

El modelo de competencias propone formar individuos integrales en los ámbitos personal, social, profesional y productivo a partir del programa institucional de tutorías (PIT) que abarca tres ejes: "El desarrollo académico, personal, y profesional, para estimular las competencias del estudiante, mejorar su práctica educativa, aprovechar sus potencialidades, desarrollar su capacidad crítica e innovadora, su desempeño escolar y apoyar su vida cotidiana" (SEP, 2013, p. 2).

\section{Definiciones}

El autoconcepto se define como la opinión que una persona tiene sobre sí misma, lo cual lleva asociado un juicio de valor e incluye la percepción de nuestras capacidades y nuestras flaquezas. Está estructurado por varios dominios: académico, personal, social y físico (Gabinet Psicológic Mataró, s. f.). El autoconcepto es muy importante en la formación de la personalidad, pues "desarrollando un autoconcepto positivo desde la adolescencia, se evitan problemas psicológicos y pedagógicos” (Luna y Molero, 2013). Estos autores hacen una clara diferenciación entre autoconcepto y autoestima: el primero lo asocian con el componente cognitivo/perceptivo (pensamientos), mientras que la autoestima la relacionan con el componente afectivo/evaluativo (sentimientos).

Los jóvenes con un autoconcepto alto tendrán mayor independencia, les será más fácil asumir responsabilidades y tendrán más resistencia a la frustración, por lo que sus relaciones con los demás serán más sanas y equilibradas. En cambio, las personas con un autoconcepto bajo difícilmente confiarán en sus competencias y habilidades personales, por lo que se sentirán infravaloradas por los demás y su forma de actuar será defensiva (Iñiguez, 2016, p. 72).

La autoestima, por otra parte, se define como la percepción que se tiene de sí mismo (Aragón, 2018, párr. 7). Esta constituye la plataforma desde la cual los individuos proyectan las diversas áreas de su vida. Se crea en la primera etapa de la vida y determina las relaciones 


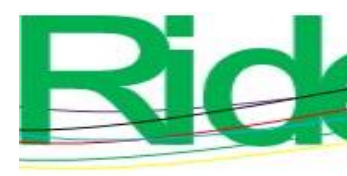

Revista Iberoamericana para la Investigación y el Desarrollo Educativo

ISSN $2007-7467$

con los demás, así como la interacción laboral y familiar. De hecho, si se cuenta con una autoestima baja, se puede modificar (Montoya y Sol, 2001) porque no es un rasgo estático ni estable en el tiempo, sino más bien un índice dinámico que se ve influido por las experiencias a las que las personas se ven expuestas (Baldwin y Hoffman, 2002, citados por Parra, Oliva y Sánchez, 2004).

La autoestima influye directamente en el comportamiento de los individuos e incide en la promoción del bienestar psicológico, pues la forma en que se evalúa a sí mismo el individuo tiene repercusión en todas las áreas del desarrollo social, emocional, intelectual, conductual y escolar. Este concepto toma relevancia porque le corresponde a la universidad favorecer la permanencia y el desarrollo académico, así como preparar profesionistas capaces de resolver con éxito problemas sociales, con responsabilidad de dirigir su vida para progresar en su carrera universitaria.

La motivación, en cambio, "es un estado interno que activa, dirige y mantiene la conducta de la persona hacia metas o fines determinados" (Gómez, 18 de octubre de 2013, párr. 2) Santrock (2002, citado por Naranjo Pereira, 2009) explica que, etimológicamente, el término motivación procede del latín motus, que se relaciona con aquello que moviliza a la persona para ejecutar una actividad. Los tres principales enfoques son los siguientes: el conductual enfatiza que las personas actúan motivadas por beneficios o recompensas; el humanista se vincula con la capacidad del ser humano para desarrollarse y su libertad de elección, y el cognitivo señala que lo que se piensa que puede ocurrir determina lo que sucede.

Dwek, Henderson, Leggett (2002, citados por Naranjo Pereira, 2009) identifican tres tipos de orientación al logro: la pericia, la incapacidad y la ejecución. Estos autores han encontrado - en el caso concreto de la población estudiantil— que los educandos muestran dos respuestas distintas ante situaciones difíciles: una de pericia o una de incapacidad. Las primeras se centran en la tarea, y no en su habilidad, por lo que disfrutan del desafío y elaboran estrategias de solución, lo cual mejora su ejecución. Las segundas se enfocan en sus insuficiencias personales, atribuyen sus dificultades a una falta de habilidad y pueden sentirse aburridas o ansiosas, lo que deteriora su ejecución. Estas personas pueden trabajar sin entusiasmo y asumen el fracaso como evidencia de su poca habilidad. 


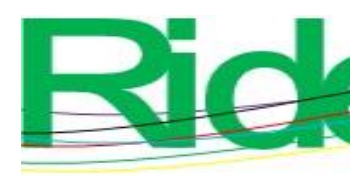

Revista Iberoamericana para la Investigación y el Desarrollo Educativo

ISSN $2007-7467$

Con respecto al desempeño académico, Cajiao (23 de mayo de 2018) lo define como la evaluación que de sus estudiantes hacen los colegios, e incorpora muchos aspectos de carácter formal e informal, como resultados académicos, comportamientos y actitudes. Por ello, la evaluación del desempeño escolar suele ser un tema muy complejo, ya que los docentes califican en el desarrollo de los estudiantes muchos factores que se entrecruzan y trascienden. Es importante tener un horizonte amplio de la evaluación que involucre el comportamiento general, así como los aspectos emocionales, intelectuales y sociales. "El éxito académico obtenido afecta el desarrollo de la autoestima y el proyecto de vida de los jóvenes y la función social que cumplirán, además, contribuye al fortalecimiento o debilitamiento de la escuela como institución social para formar a los nuevos ciudadanos" (Quintero y Orozco Vallejo, 2013, p. 3).

En el presente estudio se procura identificar los factores psicológicos con mayor influencia en el desempeño escolar de los alumnos que cursan el primero y segundo año de la carrera de Contador Público, en el Instituto Tecnológico Superior de Nuevo Casas Grandes, con el fin de elaborar estrategias de intervención para fortalecer y elevar la eficiencia terminal. Con base en ello, se formuló la siguiente hipótesis:

Ho. No existe diferencia significativa en el nivel de autoestima, autoconcepto y motivación entre los alumnos de alto y bajo desempeño académico.

H1. Existe diferencia significativa en el nivel de autoestima, autoconcepto y motivación entre los alumnos de alto y bajo desempeño académico.

\section{Materiales y métodos}

Para medir el autoconcepto se utilizó el instrumento Tennessee Self Concept Scale (TSCS) de Fitts (1972). Consta de 100 afirmaciones que pueden ser valoradas desde completamente falso (1) hasta completamente cierto (5). La validación de la escala para su aceptación y estudios posteriores, así como la autorización para efectuar la estandarización de la escala en México, su traducción y adaptación fueron hechas por Rosado en 1992. Este instrumento mide tres escalas internas (autoconcepto, autoestima y autocomportamiento), así como cinco dimensiones que constituyen al individuo (yo físico, yo moral-ético, yo personal, yo familiar y yo social) (Alfaro-García y Santiago-Negrón, 2002). 


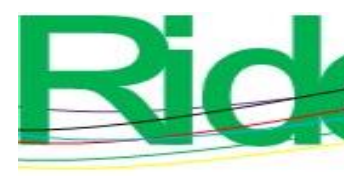

Revista Iberoamericana para la Investigación y el Desarrollo Educativo

ISSN 2007 - 7467

También se empleó como instrumento el cuestionario de autoestima de GonzálezArratia IGA 2001. Este mide la autoestima en las diferentes edades, pues está validado tanto en niños como en adultos para demostrar sus variaciones a lo largo de la vida. Consta de 25 reactivos con tres opciones de respuesta (siempre, a veces, nunca). La prueba de validez alfa de Cronbach es de .8090 y la escala está integrada por seis factores: yo, familia, fracaso, trabajo-intelectual, éxito y afecto-emocional (Camacho Fernández, Contreras Garduño y Cortés López, 2011).

La motivación se midió utilizando la escala de motivación académica (EMA) construida originalmente por Manassero y Vázquez, validada por Núñez Fontana y Pascal con un alfa de Cronbach de 0.878. Se obtiene puntaje en cinco subescalas que evalúan lo siguiente: motivación extrínseca internalizada, motivación intrínseca pura, motivación extrínseca, desmotivación y motivación intrínseca vocacional. La escala se encuentra compuesta por 28 ítems; las opciones de respuesta son presentadas por medio de una escala tipo Likert de siete puntos, donde 1 representa nada de acuerdo y 7 totalmente de acuerdo (Vanessa, 2012). Para medir el desempeño escolar se incluyó una pregunta en la encuesta que permitía al alumno escribir su promedio en el último semestre cursado. Como aspecto ético se protegió la identidad de los encuestados.

\section{Metodología}

La investigación fue de índole aplicada, ya que se procuró dar solución a una problemática. El enfoque fue cuantitativo, pues se midieron las variables autoconcepto, autoestima, motivación y desempeño académico. El diseño fue no experimental transeccional, ya que se recolectó información una sola vez, y no se manipularon variables. Se identificó una población objetivo de 78 alumnos que cursan el primero y el segundo año de la carrera de Contador Público. Para determinar la muestra se utilizó la fórmula para población finita, con un valor de confianza de $95 \%$. Se obtuvo una muestra de 43 alumnos a quienes se les administró la encuesta diseñada con tres escalas. El muestreo fue probabilístico con selección al azar. Para formar grupos iguales de hombres y mujeres se hizo un muestreo aleatorio. Se utilizó la prueba t de Student para dos muestras independientes para determinar si existían diferencias estadísticamente significativas, de acuerdo con la variable desempeño académico tomando como criterio un nivel de significancia estadística p $<0.05$. 


\section{Análisis}

Para el análisis se conformaron cuatro bloques: en el primero se compararon hombres contra mujeres para determinar la influencia de los factores analizados por género. En el segundo bloque, hombres contra mujeres (ambos con bajo rendimiento) para analizar la influencia de los factores según el género y el rendimiento académico. En el tercer bloque, hombres con bajo rendimiento contra hombres con alto rendimiento para conocer cuáles factores psicológicos tenían mayor influencia según el rendimiento académico. Por último, en el cuarto bloque, mujeres con bajo rendimiento contra mujeres con alto rendimiento para determinar cuáles factores influían en cada uno de los grupos respecto a su rendimiento académico.

\section{Resultados}

Tabla 1. Concentrado valor $p$, escala de motivación académica (EMA)

\begin{tabular}{|l|c|c|c|c|}
\hline Factor & $\begin{array}{l}\text { Hombres } \\
\text { - mujeres }\end{array}$ & $\begin{array}{l}\text { H-M ambos } \\
\text { con bajo } \\
\text { rendimient } \\
\text { o }\end{array}$ & $\begin{array}{l}\text { H bajo } \\
\text { rendimient } \\
\text { o - H con con bajo } \\
\text { alto } \\
\text { rendimient } \\
\text { o }\end{array}$ & $\begin{array}{l}\text { M } \\
\text { rendimient } \\
\text { o M con } \\
\text { alto } \\
\text { rendimient } \\
\text { o }\end{array}$ \\
\hline $\begin{array}{l}\text { Motivación } \\
\text { internalizada }\end{array}$ & 0.126 & 0.021 & 0.336 & 0.049 \\
\hline Motivación intrínseca pura & 0.237 & 0.047 & 0.438 & 0.053 \\
\hline Motivación extrínseca & 0.207 & 0.077 & 0.177 & 0.103 \\
\hline Desmotivación & 0.083 & 0.008 & 0.071 & 0.020 \\
\hline Motivación intrínseca vocacional & 0.126 & 0.012 & 0.276 & 0.026 \\
\hline
\end{tabular}

Fuente: Elaboración propia

El resultado obtenido en la tabla 1 sobre la motivación académica fue el siguiente: en el primer bloque (hombres contra mujeres) se aprecia que según el género no existe diferencia significativa; sin embargo, en el segundo bloque (hombres contra mujeres, ambos con bajo rendimiento) se observan diferencias significativas en las siguientes dimensiones: motivación extrínseca internalizada (0.021), motivación intrínseca pura (0.047), desmotivación (0.008) y motivación intrínseca vocacional (0.012); al asociarse el rendimiento académico, sí existe una diferencia significativa, por lo que estos factores sí influyen en el desempeño escolar. También se aprecia que al medir hombres con bajo rendimiento contra hombres con alto 


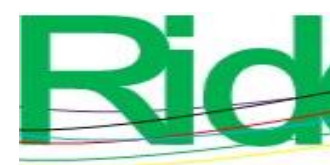

Revista Iberoamericana para la Investigación y el Desarrollo Educativo

ISSN $2007-7467$

rendimiento no se encuentra ninguna diferencia significativa (en los hombres no influye este factor). En el bloque cuatro (mujeres con bajo rendimiento contra mujeres con alto rendimiento) se aprecia diferencia significativa en motivación extrínseca internalizada (0.049), desmotivación (0.020) y motivación intrínseca vocacional (0.026). Al conseguirse diferencias significativas en los factores mencionados, se hizo una revisión de las puntuaciones obtenidas en la aplicación de la escala, y se apreciaron puntuaciones más bajas en las mujeres con un desempeño académico pobre. Por ello, se infiere que en las mujeres influye más la motivación en su desempeño académico.

Tabla 2. Concentrado valor p, instrumento González-Arriata 2001 (IGA) para medir autoestima

\begin{tabular}{|l|c|l|c|c|}
\hline Factor & $\begin{array}{l}\text { Hombres- } \\
\text { Mujeres }\end{array}$ & $\begin{array}{l}\mathrm{H}-\mathrm{M} \\
\text { ambos con } \\
\text { bajo } \\
\text { rendimiento }\end{array}$ & $\begin{array}{l}\text { H con bajo } \\
\text { rendimiento } \\
-\mathrm{H} \text { alto } \\
\text { rendimiento }\end{array}$ & $\begin{array}{l}\text { M con bajo } \\
\text { rendimiento } \\
\text { rendimiento }\end{array}$ \\
\hline Autoestima factor yo & 0.020 & 0.023 & 0.530 & 0.058 \\
\hline Autoestima factor familia & 0.186 & ---- & ---- & ---- \\
\hline Autoestima factor fracaso & 0.028 & 0.003 & 0.505 & 0.002 \\
\hline Autoestima factor trabajo intelectual & 0.047 & 0.034 & 0.194 & 0.002 \\
\hline Autoestima factor éxito & 0.112 & 0.034 & 00.728 & 0.537 \\
\hline Autoestima factor emocional & 0.025 & ---- & ---- & 0.001 \\
\hline
\end{tabular}

Fuente: Elaboración propia

Los resultados de la tabla 2 sobre la autoestima con la escala IGA demuestran una diferencia significativa en el primer bloque (hombre contra mujeres) en las siguientes subescalas: autoestima factor yo (0.020), autoestima factor fracaso (0.028), autoestima factor trabajo intelectual (0.047) y autoestima factor emocional (0.025), por lo que dicho factor tiene influencia según el género. En el segundo bloque (hombres contra mujeres, ambos con bajo rendimiento) se encontró diferencia significativa en autoestima factor yo (0.023), autoestima factor fracaso (0.003), autoestima factor trabajo intelectual (0.034) y autoestima factor éxito (0.034). Esto revela que cuando se compara el género contra el rendimiento académico los factores de autoestima tienen una influencia significativa. En el 


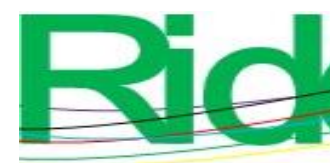

Revista Iberoamericana para la Investigación y el Desarrollo Educativo

ISSN $2007-7467$

cuarto bloque (mujeres con bajo rendimiento contra mujeres con alto rendimiento) los factores con diferencia significativa fueron autoestima factor fracaso (0.002), autoestima factor trabajo intelectual (0.002) y autoestima factor emocional (0.001). En este análisis se encontró que existe influencia de estos factores según el género y el rendimiento académico, por lo que se revisaron las puntuaciones obtenidas en las escalas y se detectó que las mujeres tienen más bajas puntuaciones en autoestima factor fracaso y autoestima factor emocional. En los hombres se aprecian bajas puntuaciones en autoestima factor yo, autoestima factor trabajo intelectual y autoestima factor éxito. Por ello, se determina que sí existe diferencia significativa según el género en la variable autoestima.

En la comparación hombres con bajo rendimiento contra hombres con alto rendimiento no existe diferencia significativa; en cambio, al comparar mujeres con alto rendimiento contra mujeres con bajo rendimiento sí existe diferencia significativa, pues se registraron puntuaciones más bajas en las mujeres con un desempeño inferior, lo que demuestra que las mujeres con autoestima baja obtienen un bajo desempeño académico. Un dato relevante hallado fue que las mujeres con alto rendimiento tienen puntuaciones más bajas en el factor trabajo intelectual, lo que indica que las mujeres con alto rendimiento se sienten inseguras en cuanto a la calidad de las tareas que realizan en sus actividades académicas.

Tabla 3. Concentrado valor p, Tennessee Self Concept Scale (subescala autoconcepto)

\begin{tabular}{|l|l|c|c|c|}
\hline Factor autoconcepto & $\begin{array}{l}\text { Hombres- } \\
\text { mujeres }\end{array}$ & $\begin{array}{l}\text { H-M ambos } \\
\text { con bajo } \\
\text { rendimiento }\end{array}$ & $\begin{array}{l}\text { H con bajo } \\
\text { rendimiento } \\
- \text { H con alto } \\
\text { rendimiento }\end{array}$ & $\begin{array}{l}\text { M con bajo } \\
\text { rendimiento } \\
\text { rendimiento }\end{array}$ \\
\hline Físico & 0.048 & 0.184 & 0.468 & 0.044 \\
\hline Moral-ético & 0.300 & 0.096 & 0.538 & 0.195 \\
\hline Personal & 0.081 & 0.055 & 0.648 & 0.052 \\
\hline Familiar & 0.774 & 0.124 & 0.236 & 0.147 \\
\hline Social & 0.337 & 0.431 & 0.532 & 0.100 \\
\hline
\end{tabular}

Fuente: Elaboración propia 


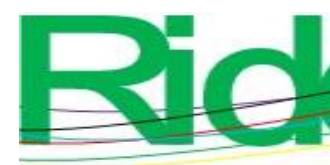

\section{Revista Iberoamericana para la Investigación y el Desarrollo Educativo ISSN 2007 - 7467}

La tabla 3 de la escala Tennessee mide el autoconcepto. En los resultados se aprecia diferencia significativa en el primer bloque (hombres contra mujeres), específicamente en el factor físico (0.048), lo que demuestra que según el género sí hay influencia de este factor psicológico. En el cuarto bloque mujeres con bajo rendimiento contra mujeres con alto rendimiento, el factor físico (0.044) indica que aparte de la diferencia significativa en el género, también influye en el rendimiento académico. Al comparar mujeres con alto rendimiento contra mujeres con bajo rendimiento sí existe diferencia significativa en el mismo factor. De hecho, al revisar las puntuaciones obtenidas en la escala, son las mujeres con rendimiento inferior quienes obtienen más baja puntuación; con este resultado se deduce que las mujeres inseguras e insatisfechas con su apariencia física obtienen menor rendimiento académico.

Tabla 4. Concentrado valor p, Tennessee Self Concept Scale (subescala autoestima)

\begin{tabular}{|l|c|c|c|c|}
\hline Factor autoestima & $\begin{array}{l}\text { Hombres- } \\
\text { mujeres }\end{array}$ & $\begin{array}{l}\mathrm{H}-\mathrm{M} \\
\text { ambos con } \\
\text { bajo } \\
\text { rendimiento }\end{array}$ & $\begin{array}{l}\mathrm{H} \text { con bajo } \\
\text { rendimiento } \\
- \text { H con alto } \\
\text { rendimiento }\end{array}$ & $\begin{array}{l}\text { M con bajo } \\
\text { rendimiento- } \\
\text { rendimiento }\end{array}$ \\
\hline Físico & 0.686 & 0.086 & 0.541 & 0.600 \\
\hline Moral-ético & 0.008 & 1.00 & 0.369 & 0.022 \\
\hline Personal & 0.038 & 0.467 & 0.519 & 0.060 \\
\hline Familiar & 0.680 & 0.103 & 0.287 & 0.226 \\
\hline Social & 0.063 & 0.466 & 0.795 & 0.101 \\
\hline
\end{tabular}

Fuente: Elaboración propia

En la tabla 4 se muestran los resultados de la escala de Tennessee para medir la autoestima. Se encontraron diferencias significativas en el bloque hombres contra mujeres en el factor moral ético (0.008) y personal (0.038). Este resultado indica que sí influye dicho factor en el género. Al analizar el cuarto bloque (mujeres con bajo rendimiento contra mujeres con alto rendimiento) se observa que existe diferencia significativa en el factor moral ético (0.022). Se demuestra así que dicho factor influye en el desempeño académico. En la revisión de la puntuación de las escalas, las mujeres con más bajo rendimiento son quienes 

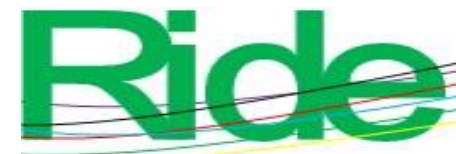

Revista Iberoamericana para la Investigación y el Desarrollo Educativo

ISSN $2007-7467$

tienen una menor satisfacción con su esquema moral o su convicción acerca de lo bueno y lo malo, así como en la aceptación de sí mismas y sus relaciones con los demás.

\section{Ecuación de Minitab (escala Tennessee)}

Por medio del modelo de regresión múltiple en el programa Minitab se obtuvo una ecuación que permite estimar la variable de respuesta desempeño académico apoyado en la variable predictora autoconcepto.

$$
\begin{aligned}
\text { Calific. Final } & =37.7+0.162 x_{1}-2.562 x_{2}+2.934 x_{3}-2.925 x_{4}+4.17 x_{5}-0.0612 x_{5}^{2} \\
& -0.0449 x_{1} x_{3}+0.0474 x_{1} x_{5}+0.0444 x_{2} x_{4}
\end{aligned}
$$

Donde

$x_{1}=$ Físico

$x_{2}=$ Moral ético

$x_{3}=$ Personal

$x_{4}=$ Familiar

$x_{5}=$ Social .

Al sustituir los valores de la ecuación con el último dato de la base de datos se obtiene:

$$
\begin{aligned}
\text { Calific. Final } & =37.7+0.162(67)-2.562(55)-2.934(70)-2.925(72) \\
& \left.+4.17(57)-0.0622(57)^{2}-0.0449(67)(70)+0.0474(67) 857\right) \\
& +0.0444(55)(72)=87.54
\end{aligned}
$$

Calificación final = 87.54 (pronosticada), la calificación real para este encuestado fue de 85 .

Con esta ecuación se puede predecir el desempeño académico con un margen muy bajo de error o variación. Esta ecuación se puede utilizar para predecir el riesgo de deserción, lo que serviría para canalizar a los alumnos y evitar en la medida de lo posible el abandono escolar, así como los índices de reprobación. 


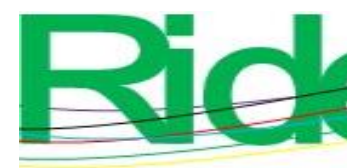

Revista Iberoamericana para la Investigación y el Desarrollo Educativo ISSN 2007 - 7467

\section{Discusión}

Los resultados de este estudio indican que factores psicológicos como la motivación, la autoestima y el autoconcepto no solo afectan a mujeres y hombres de manera diferente en cada una de sus dimensiones, sino que también influyen de manera directa en el desempeño académico. En otras palabras, los alumnos desmotivados, con una percepción pobre sobre su persona y con inseguridades son propensos a reprobar y, posteriormente, a ser parte de la estadística de deserción escolar. En concordancia con estos resultados, Rochin Berumen (2021) demuestra que los estudiantes abandonan la escuela debido a factores personales y a la falta de motivación, relaciones interpersonales, problemas psicológicos, entre otros.

Los hallazgos obtenidos, por tanto, permiten proponer como estrategia para disminuir los índices de reprobación y deserción que se apliquen estas escalas a los alumnos de nuevo ingreso y efectuar una valoración individual para reportar lo conseguido a las áreas de tutoría. De esta forma, se podría brindar ayuda a los alumnos que lo requieran para trabajar en el fortalecimiento de sus factores psicológicos. En este sentido, León Cortés (2018) hace un análisis de las principales causas de deserción con el fin de generar un modelo integral de acciones que permitan disminuir este indicador. Entre las variables menciona causas económicas, personales, de orientación, autoconcepto, desarrollo intelectual, satisfacción, entre otras, y concluye que primero se deben fortalecer los conocimientos académicos a través de talleres, posteriormente los hábitos de estudio y finalmente la autoestima.

En definitiva, los alcances del presente estudio permiten evaluar los factores psicológicos deficientes de los alumnos y su efecto en el desempeño académico; sin embargo, como limitante se debe decir que no mide los conocimientos académicos ni el ambiente dentro del cual se desarrolla el proceso enseñanza y aprendizaje, variables determinantes para el éxito escolar. Aun así, la propuesta de este trabajo representa una alternativa que se puede combinar con otras estrategias para disminuir el problema planteado. 


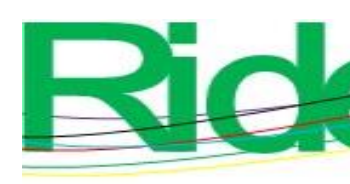

Revista Iberoamericana para la Investigación y el Desarrollo Educativo ISSN $2007-7467$

\section{Conclusiones}

Los resultados en este estudio aportan evidencia que apoya la hipótesis H1, es decir, existe diferencia significativa en el nivel de autoestima, autoconcepto y motivación entre los alumnos de alto y bajo desempeño académico. De hecho, la evaluación de las escalas aplicadas demuestra que las mujeres con bajo rendimiento están desmotivadas y tienen bajas puntuaciones en motivación extrínseca internalizada, intrínseca pura, desmotivación e intrínseca vocacional. Asimismo, cuanto más bajo es el rendimiento académico mayores son el desinterés, la apatía ante la asistencia a clases e incluso la duda acerca de continuar estudiando; en consecuencia, tendrán poca disposición para aprender, así como la negativa de adecuar su conducta a las exigencias externas, lo que provocará que tengan mayores problemas para internalizar o aceptar las normas establecidas para su conducta.

En cuanto a la autoestima, se puede indicar que las mujeres con pobre desempeño académico obtienen puntuaciones inferiores en los factores afectivo emocional, fracaso, moral ético y personal. Esto demuestra que el sentimiento de inseguridad influye negativamente, pues las hace sentir que no son importantes para nadie.

Asimismo, en lo concerniente a la escala Tennessee, en el factor físico de la dimensión autoconcepto, las mujeres con notas bajas obtuvieron puntuaciones inferiores, es decir, no les agrada su aspecto y tienen un punto de vista negativo sobre su cuerpo o apariencia.

Por otra parte, los hombres con mal rendimiento académico obtuvieron puntuaciones bajas en la escala de autoestima, específicamente en los factores trabajo intelectual, yo, éxito. De hecho, en cuanto a la ejecución del trabajo intelectual en el ambiente académico, personalmente no se valoran y se les dificulta cumplir objetivos. En cambio, los grupos con alto rendimiento académico alcanzaron mayor puntuación en cualquiera de los factores analizados. En resumen, entre más alto es el puntaje en cada una de las variables psicológicas mayor es el rendimiento académico.

La ecuación de la escala de Tennessee permite estimar el rendimiento académico basado en los totales de los cinco factores; esta información es de gran utilidad para hacer una valoración inicial a los alumnos de nuevo ingreso y determinar quiénes necesitan apoyo tanto de tutorías como del área de psicología. Al identificar los puntajes bajos, según corresponda, se puede ayudar al alumno a desarrollar los factores psicológicos que le permitan perseverar hasta conseguir sus objetivos. 


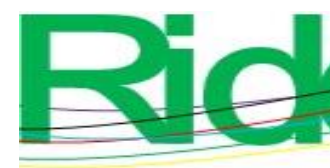

Revista Iberoamericana para la Investigación y el Desarrollo Educativo ISSN $2007-7467$

\section{Futuras líneas de investigación}

Se sugieren más estudios para comprobar los resultados obtenidos en este trabajo. Asimismo, se recomienda formular estrategias que disminuyan los factores psicológicos que influyen de forma negativa en el desempeño académico identificados en este estudio.

\section{Referencias}

Aragón, R. (14 de mayo de 2018). Autoestima. eSalud. Recuperado de https://www.esalud.com/autoestima/

Alfaro-García, R. y Santiago-Negrón, S. (2002). Estructura factorial de la Escala de Autoconcepto Tennessee. Revista Interamericana de Psicología, 36(1), 167-189.

Asociación Nacional de Universidades e Instituciones de Educación Superior (Anuies). (s. f.). Estadística de educación superior. Anuario estadístico de educación superior. Recuperado de http://www.anuies.mx/:anuies.mx/informacion-yservicios/informacion-estadistica-de-educacion-superior/anuario-estadistico-deeducacion-superior

Cajiao, F. (23 de mayo de 2018). ¿Qué es el desempeño escolar? Recuperado de https://www.magisterio.com.co/articulo/que-es-el-desempeno-escolar

Camacho Fernández, M., Contreras Garduño, J. y Cortés López, L. C. (2011). Estudio exploratorio de la autoestima de los alumnos de la Facultad de Contaduría y Administración de la Universidad Autónoma del Estado de México. Recuperado de http://www.fca.uach.mx/apcam/2014/04/07/Ponencia\%2090-UAEMex.pdf

Edel Navarro, R. (2003). El rendimiento académico: concepto, investigación y desarrollo. Revista Iberoamericana sobre Calidad, Eficacia y cambio en Educación, 1(2) Recuperado de https://www.redalyc.org/pdf/551/55110208.pdf

Gabinet Psicológic Mataró (s. f.). El autoconcepto. Recuperado de https:/gabinetpsicologicmataro.com/el-autoconcepto/

Gómez, G. R. (18 de octubre de 2013). Qué es la motivación y cómo podemos hacer para aumentarla todos los días. El Confidencial. Recuperado de https://www.elconfidencial.com/alma-corazon-vida/2013-10-18/que-es-lamotivacion-y-que-podemos-hacer-para-aumentarla-todos-los-dias_42710/ 


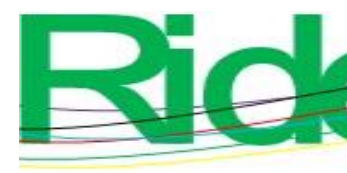

Revista Iberoamericana para la Investigación y el Desarrollo Educativo

ISSN $2007-7467$

Iñiguez, M. S. (2016). Influencia de la familia en el autoconcepto y la empatía de los adolescentes (tesis de doctorado). Universidad de Valencia: Facultad de Filosofía y Ciencias de la Educación.

León Cortés, K. (2018). Modelo de acciones para minimizar la deserción en estudiantes de educación superior en un programa de entrenamiento deportivo (tesis de maestría). Universidad Católica de Colombia: Facultad de Psicología.

Luna, N. C. y Molero, D. (2013). Revisión teórica sobre el autoconcepto y su importancia en la adolescencia. Revista Reid, (10), 43-64.

Montoya, M. Á. y Sol, C. E. (2001). Autoestima: estrategias para vivir mejor con técnicas de PNL y desarrollo humano. México, D.F: Pax México.

Naranjo Pereira, M. L. (2009). Motivación: perspectivas teóricas y algunas consideraciones de su importancia en el ámbito educativo. Educación, 33(2), 153-170.

Parra, A., Oliva, A. y Sánchez, I. (2004). Evolución y determinantes de la autoestima durante los años adolescentes. Anuario de Psicología, 35(3), 331-346.

Quintero, M. T. y Orozco Vallejo, G. M. (2013). El desempeño académico: una opción para la cualificación de las instituciones educativas. Plumilla Educativa, 12(2), 93-115.

Rochin Berumen, F. L. (2021). Deserción escolar en la educación superior en México: revisión de literatura. Revista Iberoamericana para la Investigación y el Desarrollo Educativo, 11(22), 1-11.

Román, J. A. (17 de febrero de 2020). Contrasta expansión de educación superior con baja eficiencia de alumnos. La Jornada. Recuperado de https://www.jornada.com.mx/2020/02/17/sociedad/033n1soc

Secretaría de Educación Pública [SEP] (2013). Manual del tutor del Sistema Nacional de Institutos Tecnológicos (SNIT). México: SEP.

Secretaría de Educación Pública [SEP] (2019). Principales cifras del sistema educativo nacional (2018-2019). Recuperado de https://www.planeacion.sep.gob.mx/Doc/estadistica_e_indicadores/principales_cifra s/principales_cifras_2018_2019_bolsillo.pdf

Tobón, S. (2005). Formación basada en competencias (2. ${ }^{a}$ ed.). Bogotá: Ecoe Ediciones. 


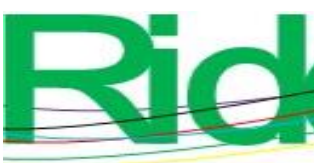

\section{Revista Iberoamericana para la Investigación y el Desarrollo Educativo ISSN 2007 - 7467}

Vanessa, J. (2012). Efecto del sexo, el nivel educativo de los padres, el aprendizaje autorregulado, la motivación al logro y la motivación académica sobre el rendimiento académico en estudiantes de la Universidad Católica Andrés Bello (tesis de licenciatura). Caracas: Universidad Católica Andrés Bello.

\begin{tabular}{|c|c|}
\hline Rol de Contribución & Autor (es) \\
\hline Conceptualización: & José Ángel Pendones Fernández \\
\hline Metodología: & $\begin{array}{l}\text { José Ángel Pendones Fernández (principal) Yolanda Flores } \\
\text { Ramírez (igual) Félix Arnoldo Durán Núñez (apoyo) } \\
\text { Germán Espino Olivas (apoyo) }\end{array}$ \\
\hline Software & José Ángel Pendones Fernández \\
\hline Validación: & $\begin{array}{l}\text { José Ángel Pendones Fernández (principal) Yolanda Flores } \\
\text { Ramírez (igual) Félix Arnoldo Durán Núñez (apoyo) }\end{array}$ \\
\hline Análisis Formal: & $\begin{array}{l}\text { José Ángel Pendones Fernández (principal) } \\
\text { Yolanda Flores Ramírez (igual) }\end{array}$ \\
\hline Investigación: & $\begin{array}{l}\text { José Ángel Pendones Fernández (principal) Yolanda Flores } \\
\text { Ramírez (igual) Germán Espino Olivas (apoyo) }\end{array}$ \\
\hline Recursos: & $\begin{array}{l}\text { José Ángel Pendones Fernández (principal) } \\
\text { Yolanda Flores Ramírez (igual) }\end{array}$ \\
\hline Curación de datos: & $\begin{array}{l}\text { José Ángel Pendones Fernández (principal) Yolanda Flores } \\
\text { Ramírez (igual) Félix Durán Nuñez (apoyo) }\end{array}$ \\
\hline $\begin{array}{l}\text { Escritura - Preparación del } \\
\text { borrador original }\end{array}$ & $\begin{array}{l}\text { José Ángel Pendones Fernández (principal) Yolanda Flores } \\
\text { Ramírez (igual) }\end{array}$ \\
\hline Escritura - Revisión y edición & $\begin{array}{l}\text { José Ángel Pendones Fernández (principal) Yolanda Flores } \\
\text { Ramírez (igual) }\end{array}$ \\
\hline Visualización: & $\begin{array}{l}\text { José Ángel Pendones Fernández (principal) Yolanda Flores } \\
\text { Ramírez (igual) Félix Arnoldo Durán Núñez (apoyo) } \\
\text { Germán Espino Olivas (apoyo) }\end{array}$ \\
\hline Supervisión: & José Ángel Pendones Fernández \\
\hline Administración de Proyectos & José Ángel Pendones Fernández \\
\hline Adquisición de fondos & $\begin{array}{l}\text { José Ángel Pendones Fernández (principal) Yolanda Flores } \\
\text { Ramírez (igual) Germán Espino Olivas (apoyo) } \\
\text { Félix Arnoldo Durán Núñez (apoyo) }\end{array}$ \\
\hline
\end{tabular}

